\title{
Frequency and clinical implications of the isolation of rare nontuberculous mycobacteria
}

\author{
Junghyun Kim¹, Moon-Woo Seong ${ }^{2}$, Eui-Chong Kim², Sung Koo Han ${ }^{1}$ and Jae-Joon Yim
}

\begin{abstract}
Background: To date, more than 125 species of nontuberculous mycobacteria (NTM) have been identified. In this study, we investigated the frequency and clinical implication of the rarely isolated NTM from respiratory specimens.

Methods: Patients with NTM isolated from their respiratory specimens between July 1, 2010 and June 31, 2012 were screened for inclusion. Rare NTM were defined as those NTM not falling within the group of eight NTM species commonly identified at our institution: Mycobacterium avium, M. intracellulare, M. abscessus, M. massiliense, M. fortuitum, M. kansasii, M. gordonae, and M. peregrinum. Clinical, radiographic and microbiological data from patients with rare NTM were reviewed and analyzed.

Results: During the study period, 73 rare NTM were isolated from the respiratory specimens of 68 patients. Among these, $M$. conceptionense was the most common (nine patients, 12.3\%). The median age of the 68 patients with rare NTM was 68 years, while 39 of the patients were male. Rare NTM were isolated only once in majority of patient (64 patients, 94.1\%). Among the four patients from whom rare NTM were isolated two or more times, only two showed radiographic aggravation caused by rare NTM during the follow-up period.

Conclusions: Most of the rarely identified NTM species were isolated from respiratory specimens only once per patient, without concomitant clinical aggravation. Clinicians could therefore observe such patients closely without invasive work-ups or treatment, provided the patients do not have decreased host immunity towards mycobacteria.
\end{abstract}

Keywords: Nontuberculous mycobacteria, Clinical manifestation

\section{Background}

Nontuberculous mycobacteria (NTM) are defined as mycobacteria other than Mycobacterium tuberculosis complex and M. leprae. Since the recognition of NTM as possible pathogens in the 1950s [1,2], the observed occurrence of NTM lung diseases has been increasing worldwide [3-5]. This may be attributed in part to improvements in microbial diagnostic tools leading to increased isolation of NTM. Additionally, the concomitant increase in susceptible hosts such as patients with underlying lung disease or an immunocompromised state may also contribute to the observed increase in NTM lung disease [5-9].

Presently, more than 125 classes of NTM species have been identified [10]. Furthermore, newly identified species of NTM are constantly reported owing to advances

\footnotetext{
* Correspondence: yimjj@snu.ac.kr

'Division of Pulmonary and Critical Care Medicine, Department of Internal Medicine, Seoul National University College of Medicine, 101 Daehak-Ro, Jongno-Gu, Seoul 110-744, Republic of Korea

Full list of author information is available at the end of the article
}

in technologies for the detection for NTM. For example, new species such as $M$. fragae and $M$. paragordonae were identified as recently as $2013[11,12]$.

Although the clinical characteristics of diseases caused by commonly isolated NTM such as $M$. avium, $M$. intracellulare or M. abscessus are well known, those caused by newly recognized and rarely isolated NTM are not yet fully understood. In the present study, we investigated the frequency of rare NTM isolation and the clinical characteristics of patients with rare NTM.

\section{Methods}

Study population

Patients from whom NTM were isolated one or more times from respiratory specimens such as sputum, bronchoscopic wash fluid and bronchoalveolar lavage (BAL) fluid between July 1, 2010 and June 31, 2012 at Seoul National University Hospital, were included in the analysis. Hence, a total of 2556 NTM isolated 
from 1373 patients during the study period were analyzed retrospectively. The Institutional Review Board of Seoul National University Hospital approved the study protocol and waived the requirement for obtaining patient consent.

\section{Identification of NTM species}

Respiratory specimens were decontaminated with $4 \%$ sodium hydroxide $(\mathrm{NaOH})$, homogenized, and concentrated by centrifugation at $3000 \times g$ for $20 \mathrm{~min}$. The processed sediments were stained using the Ziehl-Neelsen method [13]. Concentrated specimens were cultured in MGIT tubes (Becton-Dickinson and Co.; Sparks, MD, USA) as well as in $3 \%$ Ogawa medium and observed weekly for 6 or 9 weeks after inoculation, respectively. Once cultured, M. tuberculosis and NTM were differentiated using the Gen-Probe ${ }^{\bullet}$ method (Gen-Probe; San Diego, CA, USA) [14]. Following isolation of a suspected mycobacterial species, confirmation of NTM was performed by analyzing the sequences of three genes: $16 \mathrm{~S}$ $r R N A, r p o B$, and tuf. Polymerase chain reaction (PCR) and subsequent sequence were performed and the resulting sequences were compared with those in the reference database using the basic local alignment search tool (BLAST). Mycobacterial species were identified using the 16S rRNA sequences and the algorithm described in the Clinical and Laboratory Standards Institute guidelines MM18-A [15].

\section{Definition of rare NTM}

For the purposes of this study, 'rare NTM' were defined as NTM species other than $M$. avium, $M$. intracellulare, M. abscessus, M. massiliense, M. fortuitum, M. kansasii, M. gordonae and $M$. peregrinum, which are the eight NTM species commonly identified at our institution.

\section{Clinical and radiographic characteristics}

Demographic, clinical and radiographic data of the patients from whom rare NTM were isolated were reviewed. Demographic data including age, gender, and smoking habits; past medical history of tuberculosis (TB), measles, pertussis, and sinusitis; comorbidities including malignancy, diabetes mellitus, cerebrovascular disease, rheumatic disease, inflammatory bowel disease, gastroesophageal reflux disease, and underlying lung disease; clinical data for self-reported symptoms; and findings of the physical examinations, were all thoroughly reviewed. The characteristics and distribution of lung lesions were analyzed based on chest computed tomography (CT) by two pulmonologists (J.K. and J.J.Y.), who were aware of the patients' NTM results. Patients from whom rare NTM were isolated more than once were analyzed separately.

\section{Results}

Common NTM isolated from respiratory specimens during the study period

During the study period, 2554 NTM were isolated from 1373 patients. Of these, 803 NTM were identified at the species level. Among these, 730 (90.9\%) were commonly isolated NTM species while the others (73 specimens, 9.1\%) were rare NTM. M. avium (250 specimens, 34.2\%) and $M$. intracellulare (252 specimens, $34.5 \%$ ) were the most frequently identified rare NTM species, followed by $M$. abscessus (93 specimens, $12.7 \%$; see Table 1 ).

\section{Rare NTM isolated during the study period}

During the study period, 73 NTM isolated from 68 patients were identified as rare NTM. Of those isolated, 70 (95.9\%) were from sputum and three (4.1\%) were from bronchoscopic specimens such as bronchial washsing and bronchoalveolar lavage. Among the identified rare NTM, M. conceptionense was the most common (nine specimens, $12.3 \%$ ), followed by $M$. chelonae, M. lentiflavum and M. mageritense (seven specimens each, 9.6\% respectively; Table 2). Rare NTM were isolated only once from 64/68 patients, twice from three patients, and three times from one patient. Among the patients from whom rare NTM was isolated only once, commonly isolated NTM was also isolated 2.4 times from 8.2 samples of sputum on average.

\section{Characteristics of the patient cohort from whom rare NTM were isolated}

The median age of the 68 patients from whom rare NTM were isolated was 68 years (range, 30-84 years), while 39 (57.4\%) were male. Of these patients, 24 (35.3\%) had a previous history of TB treatment. The most common underlying diseases were solid organ malignancies including lung cancer (seven patients, 10.3\%), and diabetes

Table 1 NTM commonly isolated from respiratory specimens during the study period

\begin{tabular}{ll}
\hline Species & N (\%) \\
\hline $\begin{array}{l}\text { Mycobacterium avium complex } \\
\quad \text { Mycobacterium avium }\end{array}$ & $250(34.2)$ \\
Mycobacterium intracellulare & $252(34.5)$ \\
Mycobacterium abscessus complex & \\
$\quad$ Mycobacterium abscessus & $93(12.7)$ \\
$\quad$ Mycobacterium massiliense & $53(7.3)$ \\
Mycobacterium fortuitum & $28(3.8)$ \\
Mycobacterium gordonae & $19(2.6)$ \\
Mycobacterium kansasii & $18(2.5)$ \\
Mycobacterium peregrinum & $17(2.4)$ \\
Total & $730(100.0)$ \\
\hline
\end{tabular}


Table 2 Rare NTM species isolated from respiratory specimens during the study period

\begin{tabular}{ll}
\hline Species & N (\%) \\
\hline Mycobacterium conceptionense & $9(12.3)$ \\
Mycobacterium chelonae & $7(9.6)$ \\
Mycobacterium lentiflavum & $7(9.6)$ \\
Mycobacterium mageritense* & $7(9.6)$ \\
Mycobacterium chimaerat & $6(8.2)$ \\
Mycobacterium terrae & $5(6.8)$ \\
Mycobacterium kumamotonense & $4(5.5)$ \\
Mycobacterium porcinum & $3(4.1)$ \\
Mycobacterium goodie & $2(2.7)$ \\
Mycobacterium nebraskense & $2(2.7)$ \\
Mycobacterium phocaicum* & $2(2.7)$ \\
Mycobacterium septicum & $2(2.7)$ \\
Mycobacterium celatum & $1(1.4)$ \\
Mycobacterium holsaticum & $1(1.4)$ \\
Mycobacterium senuense & $1(1.4)$ \\
Mycobacterium arupense & $1(1.4)$ \\
Mycobacterium kubicae & $1(1.4)$ \\
Mycobacterium neoaurum & $1(1.4)$ \\
Mycobacterium xenopi & $1(1.4)$ \\
Failed species identification $\neq$ & $10(13.7)$ \\
& $73(100.0)$ \\
\hline
\end{tabular}

* $M$. mageritense and $M$. phocaicum were isolated from a single patient. $\dagger M$. chimaera was isolated three times from a single patient. fldentification was attempted but failed at species level.

mellitus (six patients, 8.8\%). TNF- $\alpha$ inhibitors were used in two patients and HIV infection was confirmed in one patient. Sputum production (39 patients, 57.4\%) and coughing (30 patients, $44.1 \%$ ) were the most common selfreported symptoms. Postnasal drip (10 patients, 14.7\%) and crackles (four patients, 5.8\%) were the most common findings during physical examination (Table 3).

Upper lobes of lung were commonly involved (right upper lobe in 40 patients and left upper lobe in 33 patients). Bilateral lesions were found in 30 patients (44.1\%). In 21 (30.9\%) patients, three or more lobes were involved. Multiple nodules were the most commonly observed radiographic finding (42 patients, 61.8\%). Bronchiectasis (24 patients, 35.3\%) and cavities (13 patients, $19.1 \%$ ) were also commonly observed in radiographic examinations (Table 4).

\section{Clinical course of four patients from whom rare NTM} were isolated more than once

Four patients from whom rare NTM were isolated two or more times had follow-ups for a median duration of 378 days (range, 294-477 days). Initial chest CT showed
Table 3 Demographics and clinical characteristics of the patient cohort from whom rare NTM were isolated

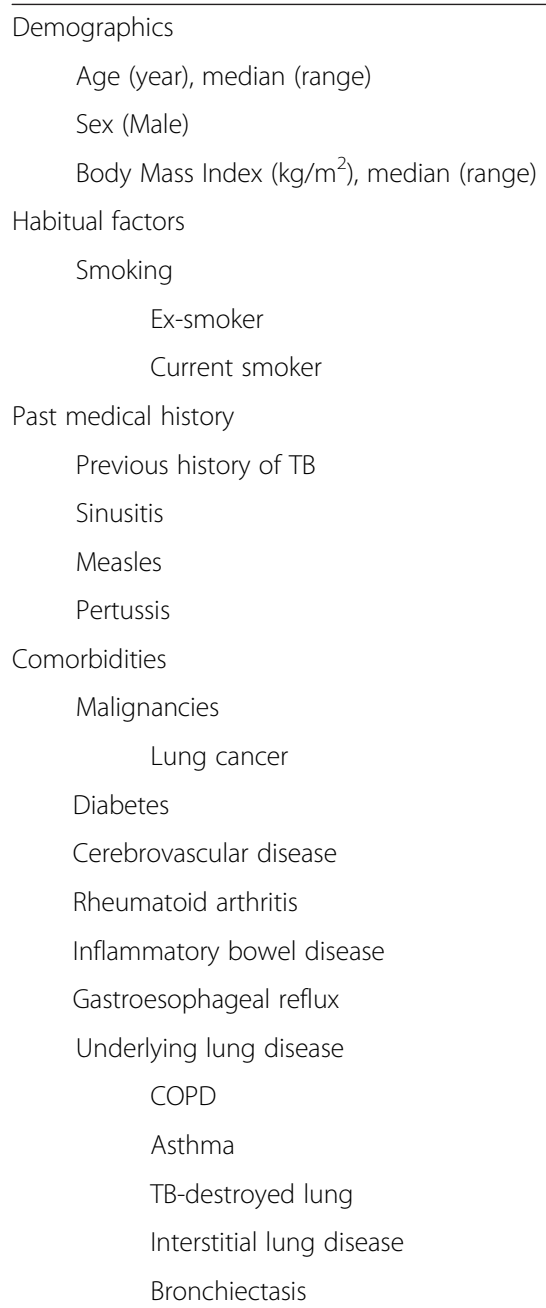

$\mathrm{N}(\%)$

$68.0(30.0-84.0)$

$39 / 68(57.4)$

$22.4(15.9-29.0)$

N (\%)

19/68 (33.8)

13/68 (22.4)

6/68 (10.3)

N (\%)

24/68 (35.3)

9/68 (13.2)

$3 / 68(4.4)$

$1 / 68(1.5)$

N (\%)

7/68 (10.3)

$1 / 68(1.5)$

6/68 (8.8)

2/68 (2.9)

$3 / 68(4.4)$

0/68 (-)

0/68 (-)

4/68 (5.9)

$4 / 68(5.9)$

3/68 (4.4)

1/68 (1.5)

23/68 (33.8)

HIV infection

1/68 (1.5)

Post-transplantation status $\quad$ 0/68 (-)

Symptoms

$\mathrm{N}(\%)$

Cough

$30 / 68(44.1)$

Dyspnea

$13 / 68(19.1)$

Hemoptysis

$11 / 68(16.2)$

Sputum

$39 / 68(57.4)$

Fever

$6 / 68(8.8)$

Myalgia

$4 / 68(5.9)$

Weight loss

$6 / 68(8.8)$

N (\%)

10/68 (14.7)

$4 / 68$ (5.8)

1/68 (1.5)

0/68 (-)

0/68 (-)

0/68 (-) 
Table 3 Demographics and clinical characteristics of the patient cohort from whom rare NTM were isolated (Continued)

Drug use

$\begin{array}{ll}\text { TNF-alpha inhibitor }^{*} & 1 / 68(1.5) \\ \text { Steroid (5-10 mg daily) }^{*} & 3 / 68(4.4) \\ \text { Other immunomodulatory drugs }^{*} & 2 / 68(2.9)\end{array}$

"All these drugs were used for the treatment of patients with rheumatoid arthritis.

patterns of bronchiectasis (patient 3), small nodules (patients 1, 3 and 4), consolidations (patients 2 and 4), and cavities (patient 4). In one patient (patient 2 in Table 5) from whom rare NTM as well as M. abscessus was isolated, the respiratory symptoms were aggravated and radiographic lesions progressed on follow-up chest CT. The clinician who managed this patient judged that this progression was caused by $M$. abscessus rather than $M$. goodie. Subsequently, a clarithromycin-based regimen was initiated for this patient. Negative conversion of sputum culture was not however achieved with treatment despite a slight symptomatic improvement. In the other two patients (patients 1 and 3 in Table 5), neither aggravation of symptoms nor progression of radiographic lesions on chest radiography occurred. These patients were observed

Table 4 Radiographic findings in patient cohort with rare NTM

\begin{tabular}{ll}
\hline & N (\%) \\
\hline Lesion location & \\
Right upper lobe & $40 / 68(58.8)$ \\
Right middle lobe & $21 / 68(30.9)$ \\
$\quad$ Right lower lobe & $22 / 68(32.4)$ \\
Left upper lobe & $33 / 68(48.5)$ \\
Left lower lobe & $21 / 68(30.9)$ \\
Lesion distribution & \\
Bilateral & $30 / 68(44.1)$ \\
Multilobar ( $\geq 3$ lobes with abnormalities) & $21 / 68(30.9)$ \\
Lesion characteristics & \\
Multiple nodules & $42 / 68(61.8)$ \\
Bronchiectasis & $24 / 68(35.3)$ \\
Cavity & $13 / 68(19.1)$ \\
$\quad$ Unilateral & $9 / 68(13.2)$ \\
$\quad$ Bilateral & $4 / 68(5.9)$ \\
Radiographic classification & \\
Upper lobe cavitary pattern & $12 / 68(17.6)$ \\
Nodular bronchiectatic pattern & $17 / 68(25.0)$ \\
Unclassifiable & $39 / 68(57.4)$ \\
\hline
\end{tabular}

without concomitant anti-NTM treatment. The other patient from whom M. chimaera was isolated at three separate time points (patient 4 in Table 5) developed new centrilobular nodules. However, this patient's other symptoms did not change and no treatment was applied (Table 5).

\section{Discussion}

New NTM are constantly reported, while the lung diseases arising from these new organisms are reported at a similarly rapid rate [16-19]. Consequently, clinicians inevitably encounter patients who present with unfamiliar and rarely identified NTM.

In the present study, various species of rarely identified NTM were isolated from respiratory specimens. $M$. conceptionense was the most frequently isolated rare NTM. This species was reported to cause infections of the skin and subcutaneous fat following surgical procedures in immunocompetent patients [20-22]. Moreover, another study demonstrated that this NTM may be a lung pathogen [18]. However, in our study, for the patient from whom $M$. conceptionense was isolated at two separate time points, neither aggravation of the symptoms nor progression of radiographic lesions was identified.

Other rare NTM species isolated from patients in the present study ( $M$. lentiflavum, $M$. mageritense, $M$. chimaera and $M$. xenopi) may also cause lung diseases $[16,18,19,23]$. However, yet other rare NTM isolated from these patients, such as M. kumamotonense and M. celatum, have generally been considered to be misidentifications or a result of culture contamination, and are thus deemed to be clinically non-pathogenic organisms [24,25].

NTM classified as rarely isolated NTM in this study may prove common in other geographic regions. For example, $M$. xenopi is commonly identified in southern Ontario, South East England and Europe [26,27], but rarely in Australia, South America, USA and Asia [28,29]. However, in this study performed in South Korea, $M$. xenopi was isolated only once.

Despite their pathogenic potential, among the patients from whom rare NTM were identified, rare NTM were isolated only once in the majority of patients $(64 / 68)$. Once-off isolation of the majority of the rare NTM suggests limited clinical significance of these NTM. Additionally, the observation that only one of the three patients from whom rare NTM were isolated two or more times without co-infection with common NTM showed evidence of radiographic aggravation, further substantiates the minimal clinical significance of rare NTM.

The limited clinical importance of the frequent onetime isolation of rare NTM presented in this study underscores the importance of the current ATS/IDSA diagnostic guidelines, which require repeated isolation of 
Table 5 Clinical characteristics of four patients from whom rare NTM were isolated more than once

\begin{tabular}{|c|c|c|c|c|c|c|}
\hline $\begin{array}{l}\text { Patients } \\
\text { (sex/age) }\end{array}$ & $\begin{array}{l}\text { Chief } \\
\text { complaint }\end{array}$ & Underlying diseases & CT finding & $\begin{array}{l}\text { Isolated NTM (number } \\
\text { of times isolated) }\end{array}$ & $\begin{array}{l}\text { Progression of } \\
\text { radiographic lesions }\end{array}$ & Treatment (Y/N) \\
\hline $1(F / 74)$ & Sputum & $\begin{array}{l}\text { - History of } \\
\text { tuberculous cervical } \\
\text { lymphadenitis }\end{array}$ & $\begin{array}{l}\text { - Nodules and } \\
\text { subsegmental } \\
\text { atelectasis }\end{array}$ & M. conceptionense (2) & - Not definite & No \\
\hline \multirow[t]{4}{*}{$2(F / 33)$} & Cough & • Undergoing & \multirow{4}{*}{$\begin{array}{l}\text { - Consolidations } \\
\text { and branching } \\
\text { opacities }\end{array}$} & M. abscessus (2) & \multirow{4}{*}{$\begin{array}{l}\text { - Increase in number } \\
\text { of multiple } \\
\text { centrilobular nodules } \\
\text { and extent of } \\
\text { bronchiectasis }\end{array}$} & Yes \\
\hline & Sputum & $\begin{array}{l}\text { Jejunostomy (due } \\
\text { to lye ingestion) }\end{array}$ & & & & \\
\hline & \multirow[t]{2}{*}{ Weight loss } & \multirow[t]{2}{*}{ - Bipolar I disorder } & & M. goodii (2) & & $\begin{array}{l}\text { Clarithromycin, } \\
\text { Rifampin, Ethambutol, } \\
\text { Moxifloxacin }\end{array}$ \\
\hline & & & & & & (Sep. 2007-Oct. 2009) \\
\hline \multirow[t]{2}{*}{$3(F / 63)$} & \multirow{2}{*}{$\begin{array}{l}\text { Bloody } \\
\text { sputum }\end{array}$} & - Diabetes & \multirow{2}{*}{$\begin{array}{l}\text { - Bronchiectasis } \\
\text { and nodules }\end{array}$} & M. phocaicum (1) & \multirow[t]{2}{*}{ - Not definite } & \multirow[t]{2}{*}{ No } \\
\hline & & $\begin{array}{l}\text { - Coronary artery } \\
\text { disease }\end{array}$ & & M. mageritense (1) & & \\
\hline \multirow[t]{2}{*}{$4(M / 59)$} & Cough & \multirow{2}{*}{$\begin{array}{l}\text { - History of pulmonary } \\
\text { TB }\end{array}$} & \multirow{2}{*}{$\begin{array}{l}\text { - Nodules, } \\
\text { consolidations, } \\
\text { fluid-containing } \\
\text { cavity }\end{array}$} & \multirow[t]{2}{*}{ M. chimaera (3) } & \multirow{2}{*}{$\begin{array}{l}\text { - New centrilobular } \\
\text { nodules and patchy } \\
\text { consolidation }\end{array}$} & \multirow[t]{2}{*}{ No } \\
\hline & Hemoptysis & & & & & \\
\hline
\end{tabular}

an NTM in the appropriate clinical setting [13]. Clinicians confronted with rare NTM could observe the patients for a while watching whether the same species of NTM would be isolated again or not.

To fully appreciate these results, a limitation of this study should be noted: species level identification was not performed on all NTM isolated from respiratory specimens. A prospective study including species identification of all NTM isolated during the certain period would likely confirm the findings of the current study.

\section{Conclusions}

Rarely identified NTM isolated from respiratory specimens have limited clinical importance in most cases. Clinicians who treat patients with rarely identified NTM could therefore observe them closely without any intensive work-ups or treatment being required, provided that these patients do not present with decreased host immunity towards mycobacteria.

\section{Abbreviations}

NTM: Nontuberculous mycobacteria; BAL: Bronchoalveolar lavage;

TB: Tuberculosis; CT: Computed tomography.

\section{Competing interests}

The authors declare that they have no competing interests.

\section{Authors' contributions}

Study concept and design: JJY. Build-up database: JK. Analysis and interpretation of data: JK, SKH, JJY. Manuscript: JK. Critical revision of the manuscript for important intellectual content: MWS, ECK. Statistical analysis: JK. Study supervisor: JJY. All authors read and approved the final manuscript.

\section{Acknowledgements}

This work was supported by grant number 800-20120025 from the Seoul National University College of Medicine (Seoul, Republic of Korea). The funders had no role in study design, data collection and analysis, decision to publish, or preparation of the manuscript.

\section{Author details}

${ }^{1}$ Division of Pulmonary and Critical Care Medicine, Department of Internal Medicine, Seoul National University College of Medicine, 101 Daehak-Ro, Jongno-Gu, Seoul 110-744, Republic of Korea. ${ }^{2}$ Department of Laboratory Medicine, Seoul National University College of Medicine, 101 Daehak-Ro, Jongno-Gu, Seoul 110-744, Republic of Korea.

Received: 11 July 2014 Accepted: 23 December 2014

Published online: 09 January 2015

References

1. Tsukamura M, Kita N, Shimoide H, Arakawa H, Kuze A. Studies on the epidemiology of nontuberculous mycobacteriosis in Japan. Am Rev Respir Dis. 1988;137:1280-4

2. O'Brien RJ, Geiter LJ, Snider DE. The epidemiology of nontuberculous mycobacterial diseases in the United States: results from a national survey. Am Rev Respir Dis. 1987;135:1007-14.

3. Henry MT, Inamdar LO, Riordain D, Schweiger M, Watson JP. Nontuberculous mycobacteria in non-HIV patients: epidemiology, treatment and response. Eur Respir J. 2004;23:741-6.

4. Martin-Casabona N, Bahrmand AR, Bennedsen J, Thomsen VO, Curcio M, Fauville-Dufaux $\mathrm{M}$, et al. Non-tuberculous mycobacteria: patterns of isolation; a multicountry retrospective survey. Int J Tuberc Lung Dis. 2004:8:1186-93.

5. Wolinsky E. Nontuberculous mycobacteria and associated diseases. Am Rev Respir Dis. 1979;119:107-59.

6. Falkinham 3rd JO. Ecology of nontuberculous mycobacteria-where do human infections come from? Semin Respir Crit Care Med. 2013;34:95-102.

7. Jouanguy E, Altare F, Lamhamedi S, Revy P, Emile JF, Newport M, et al Interferon-r receptor deficiency in an infant with fatal bacille CalmetteGuerin infection. N Engl J Med. 1996;335:1956-61.

8. Dorman SE, Holland SM. Interferon-r and interleukin-12 pathway defects and human disease. Cytokine Growth Factor Rev. 2000;11:321-33.

9. Holland SM. Immune deficiency presenting as mycobacterial infection. Clin Rev Immunol. 2001;20:121-37.

10. McNabb A, Eisler D, Adie K, Amos M, Rodrigues M, Stephens G, et al. Assessment of Partial Sequencing of the 65-Kilodalton Heat Shock Protein Gene (hsp65) for Routine Identification of Mycobacterium Species Isolated from Clinical Sources. J Clin Microbiol. 2004:42:3000-11.

11. Ramos JP, Campos CE, Caldas PC, Ferreira NV, da Silva MV, Redner P, et al. Mycobacterium fragae sp. nov., a non-chromogenic species isolated from human respiratory specimens. Int J Syst Evol Microbiol. 2013;63:2583-7.

12. Kim BJ, Hong SH, Kook YH, Kim BJ. Mycobacterium paragordonae sp. nov., a slowly growing, scotochromogenic species closely related to Mycobacterium gordonae. Int J Syst Evol Microbiol. 2014;64:39-45. 
13. Griffith D, Aksamit T, Brown-Elliott B, Catanzaro A, Daley CL, Gordin F, et al. An official ATS/IDSA statement: diagnosis, treatment, and prevention of nontuberculous mycobacterial diseases. Am J Respir Crit Care Med. 2007;175:367-416.

14. Bergmann JS, Yuoh G, Fish G, Wood GL. Clinical evaluation of the enhanced Gen-Probe Amplifi ed Mycobacterium Tuberculosis Direct Test for rapid diagnosis of tuberculosis in prison inmates. J Clin Microbiol. 1999;37:1419-25.

15. Performance standards for antimicrobial susceptibility testing; 18th informational supplement. In Book Performance standards for antimicrobial susceptibility testing; 18th informational supplement (Editor ed.^eds.). pp. M100-S118. City: Clinical and Laboratory Standards Institute; 2008:M100-S118.

16. Cohen-Bacrie S, David M, Stremler N, Dubus JC, Rolain JM, Drancourt M. Mycobacterium chimaera pulmonary infection complicating cystic fibrosis: a case report. J Med Case Rep. 2011;5:473.

17. Martinez-Gonzalez DFJ, Navarro-Ortega D, Muñoz C, Martí-Obiol R, Borrás-Salvador R. Achalasia and mycobacterium goodii pulmonary infection. Pediatr Infect Dis J. 2011;30:447-8.

18. Kim SY, Kim MS, Chang HE, Yim JJ, Lee JH, Song SH, et al. Pulmonary Infection Caused by Mycobacterium conceptionense. Emerg Infect Dis. 2012:1:174-6.

19. Jeong BH, Song JU, Kim W, Han SG, Ko Y, Song J, et al. Nontuberculous mycobacterial lung disease caused by Mycobacterium lentiflavumin a patient with bronchiectasis. Tuberc Respir Dis. 2013;74:187.

20. Liao CH, Lai CC, Huang YT, Chou CH, Hsu HL, Hsueh PR. Subcutaneous abscess caused by Mycobacterium conceptionense in an immunocompetent patient. J Infect. 2009;58:308-9.

21. Yang HJ, Yim HW, Lee MY, Ko KS, Yoon HJ. Mycobacterium conceptionense infection complicating face rejuvenation with fat grafting. J Med Microbiol. 2011;60:371-4

22. Lee KH, Heo ST, Choi SW, Park da H, Kim YR, Yoo SJ. Three cases of postoperative septic arthritis caused by Mycobacterium conceptionense in the shoulder joints of immunocompetent patients. J Clin Microbiol. 2014;52:1013-5.

23. Carrillo MC, Patsios D, Wagnetz U, Jamieson F, Marras TK. Comparison of the spectrum of radiologic and clinical manifestations of pulmonary disease caused by Mycobacterium avium complex and Mycobacterium xenopi. Can Assoc Radiol J. 2014;65:207-13.

24. Rodríguez-Aranda A, Jimenez MS, Yubero J, Chaves F, Rubio-Garcia R,

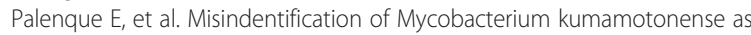
M. tuberculosis. Emerg Infect Dis. 2010;7:1178-80.

25. Tjhie JH, van Belle AF, Dessens-Kroon M, van Soolingen D. Misidentification and Diagnostic Delay Caused by a False-Positive Amplified Mycobacterium tuberculosis Direct Test in an Immunocompetent Patient with a Mycobacterium celatum Infection. J Clin Microbiol. 2001;39:2311-2.

26. Andrejak C, Thomsen VO, Johansen IS, Riis A, Benfield TL, Duhaut $P$, et al. Nontuberculous pulmonary mycobacteriosis in Denmark: incidence and prognostic factors. Am J Respir Crit Care Med. 2010;181:514-21.

27. Brode SK, Jamieson FB, Ng R, Campitelli MA, Kwong JC, Paterson JM, et al. Risk of mycobacterial infections associated with rheumatoid arthritis in ontario, Canada. Chest. 2014;146:563-72.

28. Hoefsloot W, van Ingen J, Andrejak C, Angeby K, Bauriaud R, Bemer P, et al. The geographic diversity of nontuberculous mycobacteria isolated from pulmonary samples: an NTM-NET collaborative study. Eur Respir J. 2013;42:1604-13

29. Wang HX, Yue J, Han M, Yang JH, Gao RL, Jing $\sqcup$, et al. Nontuberculous mycobacteria: susceptibility pattern and prevalence rate in Shanghai from 2005 to 2008. Chin Med J. 2010;123:184-7.

\section{Submit your next manuscript to BioMed Central and take full advantage of:}

- Convenient online submission

- Thorough peer review

- No space constraints or color figure charges

- Immediate publication on acceptance

- Inclusion in PubMed, CAS, Scopus and Google Scholar

- Research which is freely available for redistribution 